Relations industrielles

Industrial Relations

\title{
Formation syndicale
}

\section{Le Service d'éducation de la C.T.C.C.}

\section{Fernand Jolicoeur}

Volume 4, numéro 6, février 1949

URI : https://id.erudit.org/iderudit/1023493ar

DOI : https://doi.org/10.7202/1023493ar

Aller au sommaire du numéro

Éditeur(s)

Département des relations industrielles de l’Université Laval

ISSN

0034-379X (imprimé)

1703-8138 (numérique)

Découvrir la revue

Citer cet article

Jolicoeur, F. (1949). Formation syndicale : le Service d'éducation de la C.T.C.C.

Relations industrielles / Industrial Relations, 4(6), 58-59.

https://doi.org/10.7202/1023493ar

Tous droits réservés @ C Département des relations industrielles de l’Université Laval, 1949
Ce document est protégé par la loi sur le droit d'auteur. L’utilisation des services d’Érudit (y compris la reproduction) est assujettie à sa politique d'utilisation que vous pouvez consulter en ligne.

https://apropos.erudit.org/fr/usagers/politique-dutilisation/ 
dustries de base étant nationalisées, on a parlé en certains milieux de la fascisation des unions ouvrières: «The written concensus of opponents of the closed shop seems to be that the child, which has grown up to be the Government, and which soon may be the largest employer in Great Britain, will use the closed shop through the Trades Union Congress to perpetuate itself and crush all opposition. ${ }^{4}$

Mais ce débat politique ne touche pas du tout les entreprises privées où l'atelier fermé reste toujours une pratique admise par tous au point qu'on ne prend même pas la peine de l'inclure comme une clause spéciale dans les conventions collectives de travail.

(4) Jérome TONER, op. cit. page 15.

\title{
LE SERVICE D'ÉdUCATION DE LA C.T.C.C.
}

\author{
Fernand Jolicoeur
}

La Confédération des Travailleurs Catholiques du Canada groupe aujourd'hui près de $\mathbf{9 0 , 0 0 0}$ membres. Il est devenu un lieu commun de dire que c'est maintenant le mouvement ouvrier le plus représentatif dans la province de Québec. Cette expansion continuelle est due à la compétence et au dévouement de ses organisateurs et techniciens autant qu'à la valeur des principes qui l'animent.

Devant ce fait accompli et l'avenir inconnu, le problème s'est posé pour la C.T.C.C. de consolider définitivement et sûrement ses positions acquises, puis d'orienter son action vers le seul but qu'elle doit poursuivre et qui peut lui assurer la vie, celui qu'indique la doctrine sociale de l'Eglise. Afin de préciser ce but, de le faire connaitre dans tout le mouvement ouvrier, et de former ses membres à le poursuivre «par la voie la plus aisée et la plus courte», la C.T.C.C. a fondé son propre Service d'Education.

On ne peut imaginer un mouvement syndical ouvrier, surtout un mouvement d'inspiration catholique, sans organisme de formation. D'ailleurs l'histoire de la C.T.C.C. prouve amplement qu'elle n'a pas dérogé à ce principe. Dès ses débuts, nous rencontrons des cercles d'études où se sont formés les chefs qui l'ont fait naître et grandir à sa taille actuelle.

A Québec, M. l'abbé Maxime Fortin, premier aumônier de la C.T.C.C., organisait vers 1915 , ce qu'il appelait la «Commission des Questions Ouvrières de l'Action Sociale Catholique ». A Montréal, M. l'abbé Edmour Hébert fondait, en 1918, avec l'aide de l'Ecole Sociale Populaire, le fameux cercle Léon XIII, considéré comme le premier cercle d'étude syndical du mouvement. Dans chaque région de la province, ces exemples furent suivis avec fruit et, vers 1938, se formait la «Fédération des Cercles d'Etudes» de la C.T.C.C. Cette dernière a fonctionné jusqu'en 1946, alors qu'on commença à songer à un organisme plus élaboré, plus à la portée des nouveaux besoins du monde ouvrier.

On chercha une formule et on attendit d'avoir les moyens financiers voulus pour mettre sur pied un véritable Service d'Education, où un «libéré 》 pourrait diriger le travail et orienter les études sur tous les plans nécessaires. Au congrès de la C.T.C.C. à Hull, en octobre 1948, le projet longtemps caressé prit enfin corps. Le Service d'Education de la C.T.C.C. était né et un officier du mouvement entrait en charge dès le début de novembre dernier.

$$
* \quad * \quad *
$$

Pour expliquer la nature et le rôle de ce nouveau service, il faut dire d'abord que ce n'est pas une école que la C.T.C.C. a voulu fonder, école où des professeurs dispenseraient une science à des élèves. Le Service d'Education sera plutôt un centre d'études en commun. Comme le nom le dit bien, ce sera un «service», service organisé à l'intérieur du mouvement lui-même pour orienter les études, les compiler, les diffuser; service auquel contribueront tous les chefs du mouvement et dont profiteront tous les ouvriers.

Ce nouveau service syndical ne devra pas se contenter de faire l'initiation syndicale, qui s'impose cependant, ni de former des négociateurs, mais surtout d'expliquer le sens profond du syndicalisme, le pourquoi et l'orientation essentielle du mouvement ouvrier.

Or la C.T.C.C. s'appuie sur la doctrine sociale de l'Eglise pour affirmer que la structure actuelle 
de la société doit être réformée radicalement. Et elle croit que le syndicalisme ouvrier est un élément de base de cette future société.

Les cadres actuels de la société ne comprennent pas la classe ouvrière. C'est le syndicalisme qui a mission d'intégrer cette classe dans les cadres nouveaux qui s'imposent, puis de lui apprendre les droits et les devoirs qui découleront de cette nouvelle responsabilité.

Il ne s'agit donc pas simplement d'apaiser des revendications ouvrières, encore moins de tirer parti du syndicalisme pour consolider les positions actuelles de la société économique. Il ne s'agit même pas de mettre de l'avant certaines formules qui sans une force pour les contrôler, bien loin d'orienter toute la société vers la poursuite du bien commun, finiraient par transformer les ouvriers eux-mêmes en profiteurs.

Pie XII lui-même, dans son discours aux Associations catholiques des Travailleurs italiens (29 juin 1948) indique «la haute fin vers laquelle doit tendre » le mouvement ouvrier : «la formation des travailleurs vraiment chrétiens qui, excellant également en capacité dans l'exercice de leur art et en conscience religieuse, sachent mettre en harmonie la ferme protection de leurs intérêts économiques avec le sentiment le plus strict de la justice et avec la sincère volonté de collaborer avec les autres classes de la société au renouveau chré. tien de la vie sociale tout entière. »

Voilà tout le programme du Service d'Educa- tion de la C.T.C.C. C'est un véritable souffle de vie qu'il aura le devoir d'entretenir ou de communiquer. Cet organisme répondait à un besoin pressant. Le mouvement syndical catholique et national a tellement besoin de chefs convaincus, fiers de leur organisation professionnelle, éclairés tant au point de vue économique que social, « de témoins vivants d'une doctrine qui ne trompe pas », de chefs qui savent où ils vont et vers quel sommet doit s'orienter le syndicalisme !

Le Service d'Education de la C.T.C.C. se fera un devoir de collaborer avec tous ceux qui s'occupent de formation ouvrière, d'éducation des adultes, il aura besoin de l'expérience de tout le monde pour atteindre sa fin. Il existe lui-même aujourd'hui parce qu'il presse de former plus de syndicalistes militants «qui acceptent que les principes de justice et de charité chrétienne leur coûtent quelque chose », selon l'expression du secrétaire général de la C.T.C.C., M. Jean Marchand.

Le syndicalisme est un élément de réforme, non de révolte, non plus de secourisme de la socité économique actuelle. Il tend à la collaboration avec ceux qui le comprennent. Voilà le problème tel qu'il doit être posé. Il faut être du mouvement, non pour le comprendre, mais pour le sentir, non pour l'expliquer, mais pour le réaliser tout en l'expliquant. - C'est pourquoi la C.T.C.C. a voulu fonder son propre Service d'Education qui posera ce problème et participera à sa solution.

\section{PROPOS DU LECTEUR}

\section{L'avenir de la loi de la convention collective}

En toutes choses, il importe de bien préciser les principes et les faits. Dans le domaine des relations du travail, les interrelations entre ces deux termes sont d'ordinaire spécifiées et concrétisées par des lois: lois générales ou sociologiques, lois particulières ou codifiées; ces lois générales deviennent codifiées lorsqu'elles ont été préalablement confirmées par des habitus ou des faits sociaux.

La situation des relations du travail avant 1934 était plutôt primaire. Aucune législation ou mieux encore une législation précaire venait régir les activités dans ce domaine. Lorsque la loi fut adoptée, il était évident qu'elle avait pour but principal de propager la convention collective à extension juridique. Des faits précis avaient amené l'application d'une telle mesure.

Le législateur s'était rendu compte, d'abord, que la concurrence entre des industries similaires constituait le principal obstacle à la diffusion des conventions collectives de travail. En présence d'une convention collective particulière, l'employeur énonçait l'opinion suivante :
«Vous ne pouvez m'obliger, moi, employeur particulier, à me soumettre à des conditions dont mon principal concurrent est exempté; faites signer cette convention par tous mes concurrents à la fois et en même temps, et j'accepterai moi-même la convention collective que vous me soumettez ainsi, nous serons tous sur un même pied d'égalité ».

Argument d'affaire, étant donné que le salaire est un élément constitutif du prix de revient, l'employeur n'ayant qu'à augmenter proportionnellement son prix de vente pour percevoir un profit équivalent. Mais augmenter obligatoirement un prix de vente lorsqu'un concurrent peut le laisser au mềme niveau, c'est se placer dans une position de concurrence inférieure.

La Loi de la convention collective a pour but de surmonter cet obstacle en décrétant l'extension des clauses d'une convention collective à telle branche de l'activité économique, à condition que les stipulations aient, à la discrétion du ministre, «une signification et une importance prépondérantes $\gg$.

L'intéressant article paru dans l'édition de décembre du Bulletin des relations industrielles de Laval comporte, à mon sens, un éloge trop généralisé de la Loi de la convention collective, non pas que l'éloge n'ait, en soi, sa raison d'être, mais parce que l'auteur semble vouloir faire de la dite loi une panacée universelle qui guérirait tous les maux inhérents aux relations du travail. Il s'agit bien de poser les limites sociologiques d'une telle loi: 